\title{
LES STRATÉGIES PERFORMATIVES DANS LE DISCOURS ENVIRONNEMENTALISTE
}

\author{
"Il s'agit toujours de changer le pansement \\ plutôt que de penser le changement $"{ }^{1}$
}

\section{Gino Gramaccia ${ }^{2}$}

Cette étude aborde les propriétés performatives du discours environnementaliste dans les organisations. Comment l'entreprise s'y prend-elle pour rendre des comptes sur son impact social et environnemental auprès de publics susceptibles d'être affectés par ses activités, ou, plus simplement, pour prendre les devants en matière de protection de l'environnement (Tremblay et al., 2007, pp. 218-219), ou encore, selon les événements, fournir des explications, présenter des excuses, rassurer des victimes, redorer son blason? Notre hypothèse est qu'elle y parvient en usant d'actes de langage, au sens du philosophe anglais Austin.

1 Latouche, 2007, p. 25.

2 Gino Gramaccia est professeur en Sciences de l'information et de la communication et chercheur au MICA à l'Université de Bordeaux 1. II est directeur éditorial de la revue Communication \& Organisation et président honoraire de la Société Française des Sciences de l'information et de la communication (SFSIC).

Recherches en communication, $\mathrm{n}^{\circ} 35$ (2011). 
La performativité est constitutive du statut juridique de textes de portée plus normative (normes, chartes, conventions, lois, décrets, déclarations officielles...). A quelle condition le discours environnementaliste peut-il revendiquer une telle légitimité ? A quel titre l'entreprise peut-elle revendiquer un statut d'écocitoyenneté ? Certes, et ce point constitue l'essentiel de notre propos, le performatif environnementaliste peut être employé dans diverses stratégies de langage : parodies, esquives, procédés dilatoires...

\section{Le performatif environnementaliste}

Partons de quelques rappels théoriques : Austin distingue l'acte illocutoire, qui fait ce qu'il dit par le fait de dire, de l'acte perlocutoire, qui produit un certain effet chez le destinataire. Par exemple, la conduite d'obéissance (le perlocutoire) résulte de la transformation de la relation entre l'émetteur et le destinataire produite par l'énonciation de l'ordre (l'illocutoire). La force de l'acte de discours est liée à l'usage d'une forme syntaxique élémentaire et à ses applications dérivées : le présent de l'indicatif à la première la personne du singulier. Mais, pour Austin, un énoncé performatif peut réussir ou échouer. Son succès dépend, dit-il, de conditions de félicité : ce sont, principalement, la légitimité, l'autorité, le statut du locuteur. La force de l'énoncé dépend du type d'acte qu'il vise à accomplir (promettre, avertir, conseiller...), du contexte de l'énonciation formé des paramètres de l'indexicalité (locuteur, interlocuteur, lieu et moment), de ses diverses modalités (déclarative, impérative, exclamative...). Dans cette " situation discursive totale » (selon Austin), l'acte de discours tire également sa force (ou sa « puissance d'agir », selon Butler (Butler, 2004, p.29), de sa référence à un système de conventions, de règles et de rituels communs aux interlocuteurs.

On voit tout le parti que l'on peut tirer de ces considérations pragmatiques pour l'étude de notre performatif environnementaliste dont les stratégies croisent les références scientifiques, les accents souverains du discours politique et la symbolique du récit épique. L'exemple simple est celui du slogan et de l'iconographie publicitaires. «De l'énergie avec moins de $\mathrm{CO}_{2}$ pour l'Europe », énonce EDF en quatrième de couverture de la revue Energie Magazine ${ }^{1}$. Sur fond de ciel bleu, de montagnes et

1 Energie Magazine, $\mathrm{n}^{\circ}$ 30, juillet-août 2010. Le texte complet est le suivant : « EDF 
de prairie, un enfant souffle sur des pétales qui, s'envolant, reforment la couronne d'étoiles du drapeau européen. Ce message est une évocation symbolique de la norme environnementale et une référence implicite aux arbitrages qui ont donné raison et priorité à la sagesse politique aux dépens des impératifs marchands. Ce type d'énoncé est performatif au sens où il formule un engagement industriel qui ne contredit pas les valeurs exprimées par la norme. Dans ce cas, il est important de préciser - et tel est le cas dans ce message - que la technologie et ses budgets seront « arraisonnés » par la norme. Ainsi - et nous revenons plus loin sur ce point - la domestication de la " puissance » technique devient une condition de félicité supplémentaire.

Dans le cas des grandes compagnies pétrolières - et de British Petroleum, puisque nous nous appuierons sur cet exemple - les conditions de félicité sont un peu plus complexes à saisir. Peut-on dire des responsables du groupe, lorsqu'ils affirment, en juin 2009, que BP a « la capacité de répondre, dans toute la mesure du possible, au pire cas d'écoulement ${ }^{1} »$, qu'ils incarnent de réelles conditions de félicité pour instaurer ipso facto la confiance ? Dans le cas de BP, ces énonciateurs font explicitement référence à la réputation de technicité du Groupe et moins sans doute, à une certaine image de l'industrie pétrolière passablement écornée par les catastrophes maritimes (D'Almeida, 2007, p. 115). Dans cet exemple, la technicité, l'ingénierie et l'expertise technologique sont des arguments que l'énonciateur mobilise dans un but strictement performatif. Ici, l'énoncé environnementaliste est bien plus qu'une simple anticipation de la réparation : il vaut pour réparation.

\section{L'ingénierie environnementale}

Les référentiels réglementaires de nature technique, comptable, administrative et sociétale ont pour le moins l'avantage de rendre politiquement et socialement crédibles les choix d'ingénierie dans les secteurs susceptibles d'engager la responsabilité dite " sociale » de l'entreprise. " La solution idéal-typique est alors évidente », recon-

produit et commercialise une énergie qui émet trois fois moins de $\mathrm{CO}_{2}$ que les autres grands énergéticiens, grâce au nucléaire, à l'hydraulique et aux énergies renouvelables. Chaque jour, EDF investit un million d'euros en $R \& D$ pour garder son avance auprès de ses 38 millions de clients. Vous aussi découvrez toutes les clefs pour comprendre et agir au quotidien sur www.moinsdeco2.com ».

1 Cl. Le journal Le Monde du mardi 22 juin 2009. 
naît Alain Lapointe, puisqu'il s'agit d'« adopter des normes développées à l'externe et qui bénéficient d'un capital de légitimité reconnu » (Lapointe, 2007, p. 73). C'est le cas, par exemple, de la norme $\mathrm{BBC}^{1}$ qui a inspiré la réglementation thermique des bâtiments (la RT 2012), laquelle devrait s'imposer à partir de 2011, en France, aux immeubles tertiaires, aux bâtiments publics, à toutes les constructions prévues dans les zones de rénovation urbaine et enfin, à partir du $1^{\text {er }}$ janvier 2013, aux habitations neuves. Le contenu technique et économique de la norme définit d'emblée les conditions optimales de l'entente entre toutes les parties prenantes d'une opération d'ingénierie environnementale.

La norme valide à la fois la performance technique de l'ingénieur et la décision politique qui en est à l'origine. Elle réhabilite, par la médiation d'un savoir d'ingénieur, la présence et la volonté du politique dans les projets de développement durable, en même temps qu'elle fixe un objectif éthique compris et partagé par l'ensemble des parties prenantes dans de tels projets. En fait, cette hybridation du politique, de l'organisation externe ${ }^{2}$, des techniques de l'ingénieur et de la gestion opérationnelle et stratégique de l'entreprise est le meilleur critère de succès performatif pour les énoncés politiques destinés à imposer les réglementations aux secteurs concernés ${ }^{3}$ mais également pour l'ensemble des activités de communication de l'entreprise : communication interne, relations publiques, relations presse, mécénat, publicité... Tel est le sens, pour citer d'autres exemples, de l'article 225 de la loi du 12 juillet 2010 dite Grenelle II, qui oblige les grandes entreprises à faire figurer dans leur rapport annuel des informations sur leur engagement pratique en faveur de l'environnement, et de la norme ISO 26000 qui, elle, fournit des lignes directrices très explicites sur les engagements à tenir, pour l'entreprise, en matière de responsabilité sociétale ${ }^{4}$.

1 Bâtiment basse consommation.

2 L'organisation multipartite ISO (International Standard Organisation), par exemple ou, dans le cas du label BBC, l'association Effinergie.

3 «Les référentiels ont au moins deux finalités importantes : ils guident les décisions et actions des entreprises qui les adoptent et servent de cadenas de reddition de comptes auprès des parties prenantes », Alain Lapointe, op. cit. p. 74.

4 Voir le dossier du journal Le Monde Economie, « La comptabilité vire au vert », du mardi 21 septembre 2010. 


\section{Les destinataires du performatif environnementaliste}

La question toujours soulevée est de savoir précisément à qui s'adresse le message « vert » ou, si l'on admet l'hypothèse performative, sur quel public il agit. La ligne de partage la plus simple sans doute est celle qui sépare les parties prenantes du projet socio-économique de l'entreprise (actionnaires, investisseurs, clients, fournisseurs, créanciers, institutions politiques et syndicales, salariés...) et le public nécessairement hétérogène des protagonistes diversement sensibles ou concernés par l'impact de ses activités économiques, voire de ses « externalités ». Le projet récent d'une réforme des normes comptables internationales prévoit justement d'intégrer dans les bilans d'entreprise les coûts sociaux et environnementaux (les « externalités »). Quels publics, quelles parties prenantes, quels destinataires sont concernés par cette " comptabilité durable »? Une continuité éthique est-elle concevable entre cette nouvelle gouvernance comptable et un destinataire syncrétique « en fusion » regroupant une grande variété d'acteurs sous la même exigence éthique (associations écologiques, syndicalistes, chercheurs, décideurs politiques...) ?

En vérité, le destinataire visé est un personnage à deux faces : il est à la fois citoyen globalement identifié sur le plan de l'évidence éthique et un acteur social, singulier, concret, diversement touché, selon son habitat ou sa profession, par l'impact sur l'environnement des activités polluantes, acteur que nous appellerons un gentilé ou un ethnonyme ${ }^{1}$. Le citoyen global est un personnage de l'épopée politique et civique, un actant syncrétique, à la fois destinateur, sujet-héros et destinataire identifié comme le destinataire-type des opérations de " greenwashing " pilotées par les services de communication des grandes entreprises (Libaert, 1992). Le gentilé est un interlocuteur singulier, premier destinataire, en raison de son statut de victime, effective ou potentielle, de la communication verte. Comme victime des externalités, il affiche autant d'identités sociales que de difficultés techniques, réglementaires et, au final, politiques, à résoudre.

Or les situations de crise, en marquant le retour à la gestion de l'urgence, « démembrent » le citoyen global destinataire du message éthique : la communication doit alors composer avec les gentilés ou les ethnonymes, puis avec leurs « sentinelles » (D'Almeida, 2007, p. 27),

1 Voir Wikipédia pour d'excellentes définitions de ces termes. 
lesquelles se révèlent hostiles et combatives en situation de crise. On pourrait citer les associations de consommateurs ou de riverains, les ONG environnementales, les syndicats de salariés (Audoin, Courtois, Rambaud-Paquin, 2009). Ce qui devient la communication de crise doit prendre en compte la diversité des intérêts particuliers, intégrer dans le même plan les revendications des victimes, repenser une stratégie de compensation dans un souci de réparation des dommages, minimiser dans la mesure du possible - c'est-à-dire dans les limites du recours légal - les responsabilités des fauteurs de trouble.

Mais ce que révèle surtout la crise, c'est le pouvoir silencieux des fautifs, l'accélération des logiques de profit, la dissimulation des facteurs de risque et finalement le peu d'efficacité performative des campagnes d'excuses lorsque la catastrophe survient. Celle du Golfe du Mexique en avril 2010 illustre bien ces différents points. En provoquant la marée noire, l'explosion de la plateforme a en même temps fait voler en éclats la figure syncrétique du citoyen global, utopique, imaginaire, supposé ne filtrer exclusivement que les messages conformes à l'idée du progrès.

Se sont retrouvés au cœur du débat mondial le patron de BP Tony Hayward et son successeur pressenti, Bob Dudley, les 80000 salariés, les 15 morts et les 170 blessés deux ans plus tôt de la raffinerie de Texas City, les 18 millions d'actionnaires et surtout les petits porteurs britanniques, le concurrent américain Apache, les 114000 demandes d'indemnisation, les stations-service boycottées et vandalisées, le groupe « Boycott BP » qui compte 600000 inscrits sur Facebook, le département américain de la Justice et les inculpés à venir ${ }^{1} \ldots$

Ce que révèle la crise, c'est le système (ou la « carte ») des parties prenantes ; ce que ruine la catastrophe, ce sont les conditions de l'efficacité pragmatique des discours réparateurs. Les problématiques environnementalistes conduisent les entreprises à repenser leur stratégie de légitimation en direction de publics de mieux en mieux informés et formés ou en tout cas de plus en plus hostiles, via l'action des grands médias d'information, aux pratiques du déni du risque telles qu'elles se révèlent à l'occasion de grandes catastrophes - ce que nous appelons plus loin la stratégie de forclusion et la monstrance.

1 Voir le journal Libération du 26 juillet 2010. 


\section{La monstrance et l'épars}

Nous appellerons la monstrance la mise en spectacle d'une catastrophe dans les médias de masse. Ce terme, emprunté à la liturgie catholique, désigne la pièce d'orfèvrerie ancienne destinée à exposer aux fidèles l'hostie consacrée et qui préfigure l'ostensoir. Le principe de la monstrance médiatique consiste - et ce n'est pas le moindre de son paradoxe - à détourner l'attention des citoyens (par analogie aux « fidèles ») en jouant sur la fascination qu'exerce le symbole. Nous avons deux formes de fascination : 1) celle qui inspire l'empathie, s'il s'agit d'une victime singulière, 2) celle qui inspire l'indignation ou le dégoût, s'il s'agit du persécuteur. Mais dans les deux cas, la monstrance dégrade la relation de reconnaissance en ce qu'elle inhibe ou retarde, en jouant sur l'émotion, le travail d'analyse rationnelle, alors qu'un tel travail a besoin de temps, de collectifs organisés, de paix démocratique comme autant de conditions favorables à l'échange d'idées et à l'intercompréhension.

Quant à l'épars, ce petit éclair qui n'est pas suivi d'un coup de tonnerre, nous l'employons ici pour désigner cette classe de performatifs destinés, comme la monstrance, à rassurer le citoyen global sur la sincérité des engagements des dirigeants de groupes impliqués dans une catastrophe écologique. Le rapport annuel de Total (2000) est, sur ce point, éloquent : «Pour manifester sa solidarité face aux conséquences du naufrage du pétrolier Erika, le groupe a pris des engagements importants à travers la Mission Littoral Atlantique, pour participer au nettoyage des côtes polluées, assurer les opérations de pompage de la cargaison de l'épave, éliminer les déchets et contribuer à la restauration de l'équilibre écologique des zones affectées »(Oxibar, 2009, p. 132). Une politique des épars, comme la monstrance, est une stratégie dilatoire : tout se passe comme si, avec l'épars, le fauteur de trouble s'associait à la colère du citoyen global, le grondement en moins. Ce simulacre de colère (l'éclair sans le tonnerre) lui permet de changer de statut actantiel (il devient destinateur, héros et destinataire de la mission bénéfique).

\section{L'embâcle}

Le poids politique du lobby pétrolier de BP est tel qu'il aura permis de réactiver rapidement les pouvoirs subpolitiques $(\text { Beck, 2001) })^{1}$ mis à

1 Pour Beck, les conseils d'administration des multinationales, les laboratoires de 
mal par la catastrophe, autrement dit de donner à nouveau carte blanche industrielle au même lobby sous prétexte d'enjeux socio-économiques directement liés à l'exploitation de l'énergie fossile. Moyennant quelques ajustements symboliques ou financiers comme le départ de Tony Hayward ou la vente de 30 milliards d'actifs pour faire face aux demandes de dédommagements, la production pétrolière aura pu reprendre son cours pour satisfaire les besoins du marché de l'énergie. $\mathrm{Au}$ fond, pour la subpolitique, cette production tire sa légitimité des enjeux sociaux liés à l'économie du pétrole (cette ressource est source abondante de valeur et contribue au développement de nombreux secteurs industriels). Cette forme de pensée macroéconomique, issue de l'ère industrielle classique, en intégrant fortement l'économique au social, vaut, pour ses parties prenantes, pour seule logique vertueuse de développement en dépit d' " externalités négatives » comme la marée noire. Ce modèle a largement (donc historiquement) rodé ses modes de régulation, à commencer par la procuration subpolitique, et ne doit donc pas prendre le risque d'une subversion éthique en intégrant une dimension interprétative et critique liée aux exigences environnementales. Il n'est donc pas question, pour l'institution politique comme instance de régulation bienveillante - le Congrès américain, en l'occurrence - d'interdire les forages en mer, d'instaurer une taxe carbone, de supprimer le plafonnement de la responsabilité civile ou d'augmenter le montant des pénalités en cas d'infraction aux réglementations sur la sécurité et l'environnement.

« Si BP sort de cette débâcle prospère et frétillante, où est donc passée l'idée de la responsabilité des entreprises ? ", se demande

recherche, les médias - mais aussi bien les comités « indépendants » censés soutenir les candidats politiques aux Etats-Unis et financés par les corporations géantes comme BP - sont ces instances subpolitiques au sein desquelles se forge et se met en œuvre une certaine conception du progrès et de la nouvelle société, instances se substituant ainsi, par " procuration », au pouvoir politique traditionnel. Citons par exemple p. 404 : «L'évolution technico-économique se fait donc entre la catégorie du politique et celle du non-politique. Elle devient une tierce instance, prend le statut hybride de subpolitique dans laquelle l'ampleur des transformations sociales mises en branle croît de façon inversement proportionnelle à la légitimation », et p. 472 : «Dans les débats contemporains, on n'attend plus que l'« autre société » naisse des débats parlementaires sur de nouvelles lois, mais qu'elles proviennent de l'application de la microélectronique, de la technologie génétique et des médias »... «L'élaboration de l'avenir n'est plus du ressort du Parlement ni des partis politiques, mais des laboratoires de recherche et des conseils d'administration ». 
crûment Robert Reich ${ }^{1}$. Où est passée la responsabilité politique tout court, pourrait-on ajouter, lorsqu'on observe les multiples collusions entre les multinationales et l'institution politique. Il faut admettre que, dans cet exemple, le Congrès joue le jeu de la subpolitique jusqu'au bout en limitant au maximum ses capacités d'intervention dans la sphère industrielle, lui laissant ainsi le choix des moyens stratégiques principalement financiers - dans la gestion de la catastrophe. Autrement dit, la non-intervention (voire non-ingérence) devient un mode de régulation subpolitique. Ainsi, en réponse à la débâcle, le responsable de la catastrophe et ses relais politiques vont s'efforcer d'anticiper et de contenir le flux des critiques au moyen d'une forme d'obstruction politique que nous proposons d'appeler l'embâcle, pour faire référence, au moyen d'une métaphore, à l'amoncellement de glace barrant un cours d'eau à l'époque de la débâcle. L'embâcle devient bien, dans notre contexte, une forme de construction - obstruction performative.

\section{L'évagation}

Alors que la monstrance consiste à transfigurer les conséquences sociales de la catastrophe, l'évagation est une stratégie de l'esquive, autrement dit ce procédé de discours qui consiste à détourner l'attention des observateurs critiques - et en particulier des commentateurs de presse - des affaires judiciaires, politiques ou financières dont s'emparent les médias et qui agitent 1'opinion $^{2}$ en attirant justement 1'atten-

1 Ancien secrétaire américain au travail (1992-1997), Professeur à l'Université de Californie à Berkeley. Cette citation est extraite de son article publié dans le journal Le Monde, Dimanche - lundi 9 août 2010. : « La leçon finale de BP : tous les coups sont permis. Il faut contraindre les pétroliers à agir autrement».

2 L'évagation est cette disposition de l'esprit qui le détourne des objets auxquels il devrait s'attacher. Définition proposée par le Petit Larousse Illustré, Paris / Librairie Larousse, 1914. Nous avons essayé de développer par ailleurs la notion de forclusion, comme pathologie du discours environnementaliste : "Au sens juridique, cette notion correspond à la prescription d'une action en justice et au sens psychanalytique, elle désigne, dans le cas des psychoses, l'absence de prise en compte du réel. En matière de discours environnementaliste, la forclusion est à la fois une stratégie de l'esquive au moyen d'énoncés susceptibles de sous-estimer l'ampleur d'un risque ou de minimiser l'impact d'une catastrophe et une stratégie dilatoire puisque l'esquive permet alors aux stratèges de la forclusion, en jouant sur le temps, de neutraliser recours, controverses, ultimatums et, en somme, de priver les victimes du temps nécessaire pour organiser leur défense ». Cette notion trouve une application intéressante avec le groupe BP lorsqu'il affirme, un mois 
tion de ces mêmes observateurs sur l'intention de l'énonciateur d'être reconnu comme sincère (Ambroise, 2008). Nous nous proposons de classer l'évagation dans la catégorie des performatifs de la monstrance. La monstrance politique fourmille d'exemples d'évagation discursive. En matière de communication environnementale, l'évagation est l'outil privilégié de la communication de crise - l'exemple de BP est là pour nous le rappeler. Ces actes de langage, qui rassurent sur les compétences techniques des ingénieurs ou des pompiers, communiquent en priorité sur l'intention de communiquer, moins sans doute pour " détourner l'esprit » que pour « orienter la prise de conscience » de l'importance des moyens en jeu pour gérer la catastrophe, autrement dit pour rassurer l'opinion. Dans la communication de crise, quand le contenu propositionnel porte sur les compétences et les moyens techniques, le marqueur illocutionnaire devient un outil de la stratégie de communication.

\section{Conclusion}

Le discours public sur le thème du développement durable est assurément performatif, et comme tel, il est soumis aux règles du jeu discursif du succès ou de l'échec. En l'occurrence, il échoue à l'instant précis où doivent être pris les engagements les plus sérieusement efficaces en faveur de solutions intégrées et globales : celles en faveur, par exemple, des pays pauvres, de la protection des forêts, de la baisse des émissions des gaz à effets de serre... Il prépare et affine seulement les mécanismes structurels et les procédures qui sous-tendent l'organisation des conférences (celle de Durban en Afrique du Sud, en 2011, par exemple).

Pour ne pas échouer, la communication performative doit faire explicitement référence à la preuve scientifique administrée. Dans ce cas, la communication scientifique ne souffre plus la moindre contestation dès lors que les faits scientifiques sont publiquement avérés. C'est par exemple le style choisi par Dominique Bourg lorsqu'il rappelle cette " deuxième chose que personne ne conteste, même pas les climato-sceptiques (...)», à savoir « l'importance de la composition chimique de l'atmosphère depuis le début de l'ère industrielle : $40 \%$ de plus de dioxyde de carbone depuis le début de l'ère industrielle, $20 \%$ de protoxyde d'azote en plus et un doublement de la concentration de

encore après l'explosion de la plateforme, que « l'impact de la catastrophe serait très limité ». 
méthane » (Bourg, 2010, p. 34). Ces informations scientifiques correspondraient, si l'on en revient à la distinction de Searle, au contenu propositionnel de l'acte de langage environnementaliste. La condition de félicité, qui fonde l'autorité de l'énonciateur et donne à son discours son pouvoir agissant, c'est encore une fois la preuve scientifique et ces faits que «personne ne conteste ». Quant à leur rappel systématique dans les discours fondateurs, les éditoriaux alarmistes, les professions de foi politiques, les arguments pour la formation d'un pacte social ou encore les diverses chartes pour la protection de l'environnement, ils forment le marqueur illocutionnaire qu'on peut ramener à une expression du type : « Les experts sont unanimes : la planète ne tourne plus rond. Nous sommes entrés dans une phase de changement climatique de grande ampleur et les dérèglements qu'il suscitera risquent d'être terribles $»^{1}$.

En matière de presse, le marqueur illocutoire de l'information ${ }^{2}$ intervient dans la révélation d'un scandale, le témoignage, la dénonciation, la charge. Il prend un tour performatif particulier lorsque l'enquête révèle en même temps les manœuvres qui visent à protéger des intérêts privés dans un contexte de catastrophe écologique. Sur ce point, ce titre du Journal Le Monde à propos de la marée noire en Chine de juillet 2010 est sans ambiguïté : «Marée noire : le silence des autorités chinoises. Sans l'intervention de Greenpeace, la catastrophe aurait été minimisée afin de préserver les intérêts du tourisme ».

Les communiqués de l'ONG sur l'indigence des pouvoirs publics, les photographies qui ont fait le tour du monde, les panneaux brandis par les militants pour inciter la population à porter des équipements de protection sont des actes de langage de mise en accusation des autorités et de mise en alerte des populations en danger : l'information performative a pour capacité de créer un consensus éthique sans discussion. Ce qui est dit de l'évidence éthique pourrait bien suffire à reconstituer ou à « remembrer » le citoyen global, le destinataire du Bien public mondial (BMP), et ce simplement parce qu'une telle information rend impossible, par le seul fait de son marqueur illocutionnaire, tout recours à une quelconque manœuvre dilatoire. Rien, dans ce cas, ne peut être plus longtemps différé.

1 Editorial de la revue Ecologie pratique, $n^{\circ}$ 4, juillet/août 2010.

2 Evidente dans le « J'accuse » de Zola. 


\section{Bibliographie}

Ambroise, B. (2008). Qu'est-ce qu'un acte de parole? Paris : Vrin.

Austin, J. L. (1970). Quand dire, c'est faire. Paris : Seuil.

Audoin, A., Courtois, A., \& Rambaud-Paquin, A. (2009). La communication responsable. Paris : Eyrolles.

Beck, U. (1998). La société du risque. Paris : Aubier.

Bourg, D. (2010). L'éco-scepticisme et le refus des limites. Etudes, 1-2, 29-40.

Bourg, D., Grandjean, A., \& Libaert, T. (2006). Environnement et entreprises. En finir avec les discours. Paris : Pearson Education.

Butler, J. (2004). Le pouvoir des mots. Paris : Editions Amsterdam.

Catellani, A. (2009). La communication environnementale interne d'entreprise aujourd'hui : dissémination d'un nouveau « grand récit». Communication \& Organisation, 36, 179-188.

D’Almeida, N. (2007). La société du jugement. Paris : Armand Colin.

De la Broise, P., Lamarche, T., (Eds.) (2007). Responsabilité sociale : vers une nouvelle communication des entreprises? Lille : Presses Universitaires du Septentrion.

Gardère, E., Gramaccia, G. (2004). La communication des nouvelles éthiques de l'entreprise : responsabilité sociale, développement durable, mode et design éthiques. Communication et Organisation, 26, 9-12.

Gramaccia, G. (2011). Les institutions de la médiation symbolique en quête de l'homme-trace. L'homme-trace. Paris : CNRS Editions, 273-287.

Gond, J.-P., Igalens, J (2008). La responsabilité sociale de l'entreprise. Paris : PUF.

Jalenques-Vigouroux, B. (2007). Quand les entreprises communiquent sur le développement durable : un choix libre ou contraint ? Quaderni, 64.

Lapointe, A. (2007). RSE et DD. Des pratiques en quête de légitimité. Développement durable et communications. Presses Universitaires du Québec.

Latouche, S. (2007). Petit traité de la décroissance sereine. Paris : Mille et une nuits.

Libaert, T. (1992). La communication verte. Paris : Liaisons.

Libaert, T. (2008). Communiquer dans un monde incertain. Paris : Pearson.

Libaert, T. D'Almeida, N. (2007). La communication interne de l'entreprise. Paris : Dunod.

Oxibar, B. (2009). Communication sociétale. Paris : L'Harmattan.

Pascual Espuny, C. (2008). Comment les organisations se saisissent-elles de 1' 'image verte'? Communication et Organisation, 34, 39-52.

Tremblay, S. (2007). Déclaration des communicateurs et des professionnels en relations publiques du Québec à l'égard du développement durable. Développement durable et communications, Presses de l'Université du Québec. 